5. Hegr, G.: Illustrierte Flora von Mitteleuropa. München 1906-1929.

6. JEGEL, A.: Altfrankens Imkerei in ihrem kulturgeschichtlichen Zusammenhang. In: Forstw. Centralbl., Berlin 1940.

7. Mummenнioff, E.: Nürnbergs Ursprung und Alter. Nürnberg 1908.

8. NiETSCH, H.: Wald und Siedlung im vorgeschichtlichen Mitteleuropa. Leipzig 1939.

9. SсHwaBe, E.: Die natürlichen Beziehungen des Bienenvolkes zur Eiche. Leipzig 1939.

10. Vogel, J. G.: Ab. Gottl. Schirachs Waldbienenzucht. Breslau 1774.

11. WAGLER, P.: Die Eiche in alter und neuer Zeit.

I. Progr. d. Gymn. zu Würzen 1891.

II. Berliner Studien f. Klass. Philologie und Archäologie, Berlin 1891.

12. WaLtinek, F. L.: Die vorzüglichsten in- und ausländischen Holzarten ... Bayreuth 1790.

13. Wör.CKERN, L. K.: Historia Norimbergensis Diplomatica. Nürnberg 1738.

14. ZANDER, E.: Die Zukunft der deutschen Bienenzucht. Berlin 1918.

15. Ders. und Koch, A.: Der Honig. Stuttgart 1927.

\title{
Archivalisches Material
}

\author{
S. Forstw. Centralbl. 1951, S. 746
}

\section{B U CHB E S PR E CH U N G EN}

Saat- und Pflanzschulen, in 2. Auflage herausgegeben von der Forst a bteilung der nordbadischen Landesbezirksdirektion der. Finanzen, Form $21 \times 30 \mathrm{~cm}$, 58 Seiten Text, 60 Abbildungen auf 18 Seiten Hochglanz-Bildbeilage, zu beziehen aus dem Selbstverlag der nordbadischen Landesforstabteilung Karlsruhe, Preis kart. 4,00 DM.

Die im Jahre 1938 in erster Auflage erschienene Anweisung der badischen Forstdirektion Karlsruhe, seit ihrem Erscheinen viel beachtet und zum Nutzen der forstlichen Praxis angewendet, ist in klarer, übersichtlicher Form, mustergültig mit neuen Bildern ausgestattet, in zweiter Auflage erschienen. Die seit 1938 in badischen Forstämtern gesammelten Pflanzgartenerfahrungen, aber auch die neueren wissenschaftlichen Erkenntnisse wurden mitbenutzt und haben dazu geführt, daß viele Abschnitte neugestaltet, umgearbeitet oder ergänzt wurden. Der Berichterstatter war geradezu erstaunt, wie vielfach und vielseitig keimungsphysiologische Forschungsergebnisse des Münchner Samenkundeinstitutes hier für den praktischen Pflanzgartenbetrieb nutzbar gemacht worden sind.

Ohne Weitschweiffigkeit und überflüssigen Ballast findet der Forstmann in der neuen Saat- und Pflanzschulanweisung klare Richtlinien und Vorschläge (jedoch dankenswerterweise keine Generalrezepte, da der Baumschulbetrieb sich nicht verallgemeinern läßt, sondern auch standortsbedingten Gesetzen unterliegt!) für Platzwahl und Anlage des Forstpflanzgartens, für Saatgutbeschaffung, -aufbewahrung und -vorbehandlung, Aussaat und Verschulung, Bodenbearbeitung und Düngung, Unkraut- und Schädlingsbekämpfung, Ernte (Ausheben, Transport usf.) der Forstpflanzen und die Kampbuchführung.

Die badische Pflanzschulanleitung besaß schon in ihrer ersten Auflage so viele Freunde und Benutzer, daß die verbesserte, dem jetzigen Stand der Erkenntnisse voll Rechnung tragende Neuauflage keiner weiteren Empfehlung bedarf. Sie sollte in der Hand jedes Amtsvorstandes und jedes Bezirksbeamten sein, die einen Pflanzgarten zu betreuen haben. Die Geldaufwendungen für die Beschaffung dieser Schrift lassen sich um ein Vielfaches an den Kosten des Pflanzgartenbetriebs einsparen, wenn nur einige wenige neue Vorschläge auf Grund dieser Schrift durchgeführt werden. Aber solche kaufmännisch-wirtschaftlichen Überlegungen sind leider in vielen Verwaltungen mit kameralistischer Buchführung und streng gebundenen Ausgabetiteln nicht realisierbar

ROHMEDER

Das in Heft $5 / 6$ des Forstwissenschaftlichen Centralblattes (S. 189) v. J. L. Lutz besprochene Buch von Forsting. Dr. van Dijk "Forstiche Bodenkartierungen im Keupergebiet des Strombergs in Wüttemberg" kann von der Forstdirektion Stuttgart, Marienstr. 48, zum Preis von 6,- DM bezogen werden. 